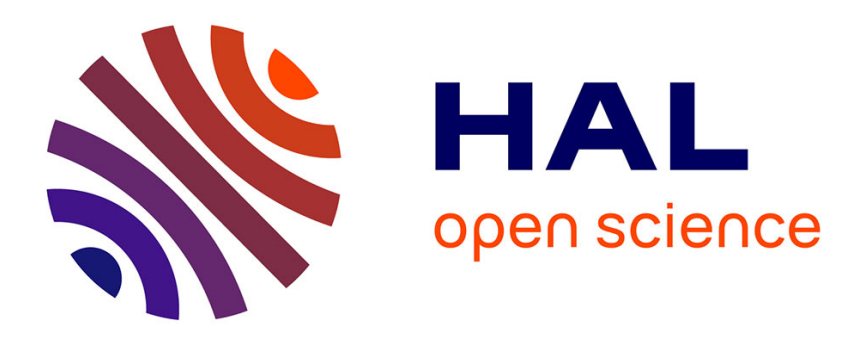

\title{
Propagation of elastic waves in a cylindrical bore containing a fluid
}

Maurice A. Biot

\section{To cite this version:}

Maurice A. Biot. Propagation of elastic waves in a cylindrical bore containing a fluid. Journal of Applied Physics, 1952, 23 (9), pp.997-1005. 10.1063/1.1702365 . hal-01368657

\section{HAL Id: hal-01368657 https://hal.science/hal-01368657}

Submitted on 21 Sep 2016

HAL is a multi-disciplinary open access archive for the deposit and dissemination of scientific research documents, whether they are published or not. The documents may come from teaching and research institutions in France or abroad, or from public or private research centers.
L'archive ouverte pluridisciplinaire HAL, est destinée au dépôt et à la diffusion de documents scientifiques de niveau recherche, publiés ou non, émanant des établissements d'enseignement et de recherche français ou étrangers, des laboratoires publics ou privés. 


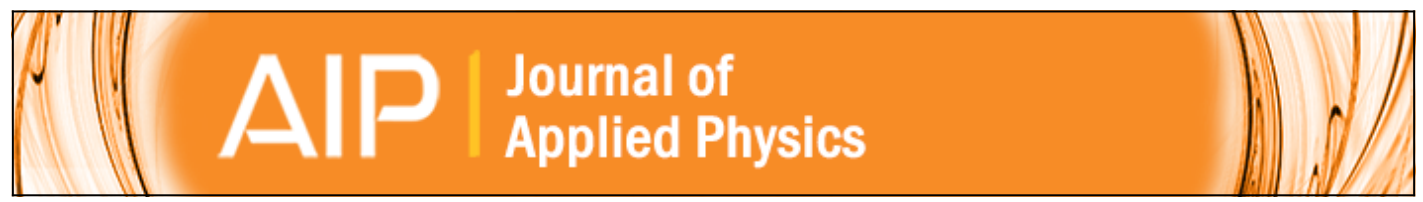

Propagation of Elastic Waves in a Cylindrical Bore Containing a Fluid

M. A. Biot

Citation: Journal of Applied Physics 23, 997 (1952); doi: 10.1063/1.1702365

View online: http://dx.doi.org/10.1063/1.1702365

View Table of Contents: http://scitation.aip.org/content/aip/journal/jap/23/9?ver=pdfcov

Published by the AIP Publishing

\section{Articles you may be interested in}

Active control of coupled wave propagation in fluid-filled elastic cylindrical shells

J. Acoust. Soc. Am. 94, 1467 (1993); 10.1121/1.408149

Active control of axisymmetric wave propagation in fluid-filled elastic cylindrical shells.

J. Acoust. Soc. Am. 90, 2271 (1991); 10.1121/1.401187

Propagation of nonlocal elastic waves in a cylindrical hole containing a fluid

J. Appl. Phys. 58, 2190 (1985); 10.1063/1.335986

Elastic Waves along a Cylindrical Bore

J. Acoust. Soc. Am. 29, 1253 (1957); 10.1121/1.1919034

Wave Propagation through Fluid Contained in a Cylindrical, Elastic Shell

J. Acoust. Soc. Am. 28, 1165 (1956); 10.1121/1.1908583

\section{High Energy Nanosecond Lasers}

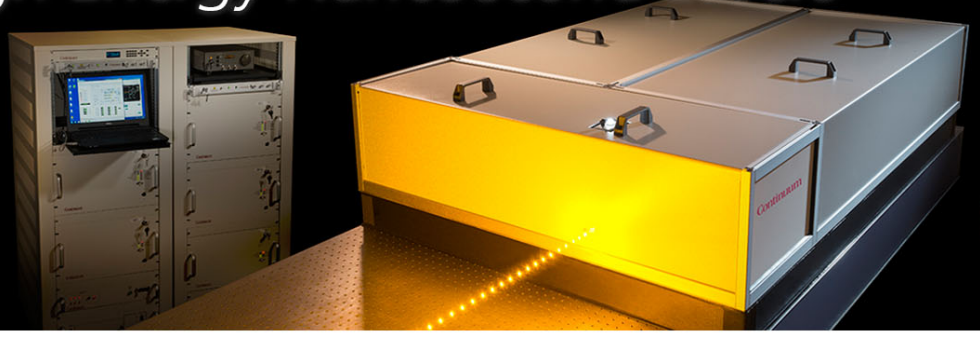


the slotted line preceding the short circuit would give rise to an extraneous reflection which would distort the standing-wave pattern, it was necessary to machine the slot flush and square with the plane of the short circuit.

The probe carriage was a narrow slab long enough to keep the slot always covered, thus minimizing contamination and evaporation of the dielectric. The ways for guiding the probe carriage along the slot were ground parallel to the center conductor within 0.0002 in. to insure constant probe depth. Spring-loaded roller bearings kept the probe carriage aligned with the center of the slot, and a micrometer screw of $1-\mathrm{mm}$ pitch, geared to a revolution counter and having an indexed head of 100 divisions, was used to drive the probe carriage along the slot. Probe displacements were discernable to $0.001 \mathrm{~mm}$ and accurate to about $0.005 \mathrm{~mm}$.

\section{Instrumental Errors}

In the measurement of $\lambda_{0}$ by means of the distance between minima of the standing wave in air, a precision of \pm 0.01 percent with comparable accuracy was consistently obtained. In nonpolar (lossless) dielectrics, the wavelength determination was only slightly less accurate. ${ }^{4}$ In medium and low loss dielectrics, $\lambda_{d}$ was measurable with an accuracy of \pm 0.1 percent, corresponding to $\mathrm{a} \pm 0.2$ percent error in the computed value of $\epsilon^{\prime}$. In high loss media, however, the accuracy was somewhat less because of the reduced probe signal amplitude which decreased the null sensitivity.

In the measurement of absorption index, the estimated attenuator error was $\pm 0.1 \mathrm{db}$, which corresponds to a numerical error of \pm 0.0018 in $\kappa$, when measured by the traveling-wave method, and to a percentage error of \pm 0.5 percent in $\log k$ or \pm 1.2 percent in $k$, when measured by the standing-wave method. In the breadth-of-the-minimum method, the error in $\kappa$ varied from 2 percent to 10 percent, depending on the magnitude of $k$. Thus, for the standing-wave method, the estimated error in the computed value of $\epsilon$ " was 1.4 percent, the errors in the other methods varying with the absorption index.

\section{ACKNOWLEDGMENT}

The author wishes to acknowledge the many helpful conversations with his colleagues on this project and their cooperation in making measurements with the apparatus. The assistance of $\mathrm{D}$. M. Olson and $\mathrm{L}$. C. Osborn in preparing the drawings is also gratefully acknowledged.

\title{
Propagation of Elastic Waves in a Cylindrical Bore Containing a Fluid
}

\author{
M. A. BIOT* \\ Columbia University, New Yark, New York
}

(Received April 7, 1952)

\begin{abstract}
The paper deals with the propagation of unattenuated elastic waves in a cylindrical bore through an elastic material of infinite extent filled with a fluid. The phase and group velocity dispersion curves are plotted for axial symmetric waves in the coupled fluid-solid system.
\end{abstract}

\section{INTRODUCTION}

A $\mathrm{N}$ analysis is here presented of the problem of propagation of elastic waves in a fluid contained in a circular bore through an elastic solid of infinite extent. Only waves of axial symmetry which are pure sinusoids along the axial direction are considered here. We are dealing essentially with the interaction between the compressible fluid and the elastic solid in the generation of propagation phenomena along the axis of the bore.

In view of the complexity of the phenomenon a clearer understanding is attained by first treating in Sec. 2 the problem of propagation of surface waves in the empty bore. In this case the surface wave, which is

\footnotetext{
* Consultant Shell Oil Company Exploration and Production Research Laboratory, Houston, Texas. The present paper is based on an unpublished Shell Oil Company report dated October $15,1949$.
}

analogous to a Rayleigh wave at the plane boundary of a semi-infinite solid, exhibits a dispersion. The phase velocity increases with the wavelength from the Rayleigh wave velocity to that of shear waves in the solid. No unattenuated waves are propagated beyond a certain wavelength at which a cutoff occurs. A family of phase and group velocity dispersion curves exists with the Poisson ratio as a parameter.

Propagation in a fluid-filled bore is taken up in Sec. 3 . The dispersion curves are first considered for the case of a fluid which is either confined in a rigid bore or suspended in free space. This corresponds to the limiting case of a fluid of zero or infinite density. In this case waves in the fluid are not affected by the elasticity of the solid. The cases of finite density are intermediate between the above, with one exception, and they correspond to interaction between fluid and solid waves. It is pointed out that not all waves result from the inter- 
action of fluid and solid waves. It is found that one branch of the dispersion curve bears no resemblance to any of the waves occurring independently in either medium but is caused solely by the interaction of the two media. This wave is designated hereafter by the appellation "Stoneley wave" because of its analogy with the waves occurring at the interface of two media. For the larger wavelengths these waves correspond to the well-known phenomenon of the water hammer in tubes. No cutoff occurs for this wave. Plots of phase and group velocity as function of wavelength to diameter ratio are presented for various combinations of the three basic parameters. These parameters are $v_{s} / c$, the ratio of shear velocity in the solid to the sound velocity in the fluid, the ratio $\rho / \rho_{1}$ of the fluid density to solid density, and Poisson's ratio $\nu$. Work by H. Lamb ${ }^{1}$ on this problem appears in a very early publication but results are given only for waves of large wavelengths. As the present analysis shows, this represents only a very limited aspect of the problem.

\section{PROPAGATION OF WAVES IN A CYLINDRICAL EMPTY BORE}

We consider a cylindrical bore of circular cross section in an elastic solid of infinite extent. This section is concerned with the case where the bore does not contain any fluid. Being a relatively simple case it is treated first, and we focus our attention on the phenomenon of propagation of axial-symmetric waves at the surface of the bore. These waves are the analog of Rayleigh waves propagating at a plane boundary. Our purpose is to determine how the propagation is affected by the

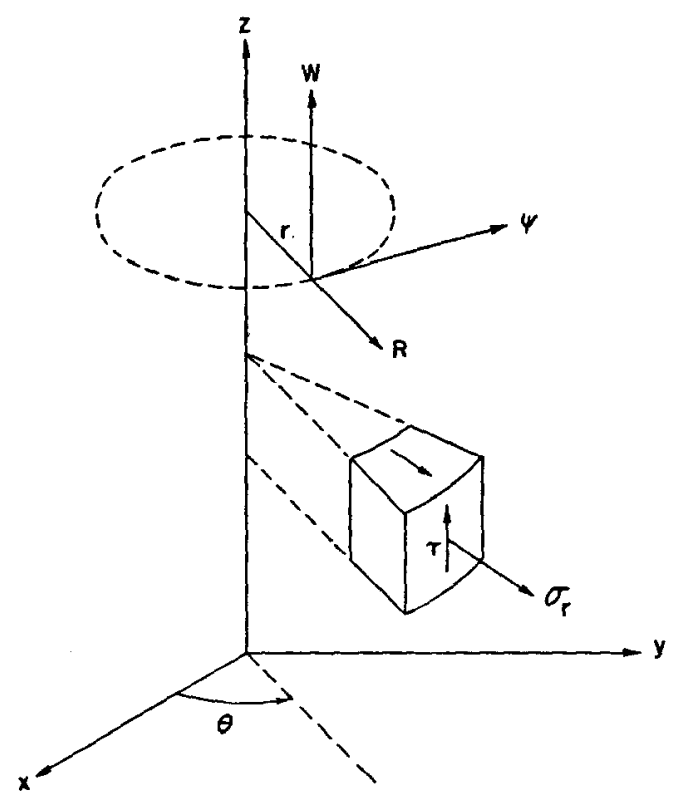

FIG. 1. Cylindrical coordinate system, $Z$ along the axis of the bore.

${ }_{1} \mathrm{H}$. Lamb, On the Velocity of Sound in a Tube as Affected by the Elasticity of the Walls, Mem. Proc. Manchester Lt. and Phil. Soc., Vol. XIII, No. 9 (1898). axial symmetric nature of the phenomenon. Because a dimension has been introduced, namely, the diameter $D$ of the bore, it must be expected that dispersion will occur; the velocity of the surface wave becomes a function of $\lambda / D$, the ratio of the wavelength $\lambda$ to the diameter.

Since we are dealing with a three-dimensional phenomenon we have three displacement components of the solid, $U, V, W$. We put

with

$$
(U, V, W)=\operatorname{grad} \phi+\operatorname{curl} \bar{\psi},
$$

$$
\begin{aligned}
& \nabla^{2} \phi=\frac{1}{v_{c}^{2}} \frac{\partial^{2} \phi}{\partial t^{2}}, \\
& \nabla^{2} \tilde{\psi}=\frac{1}{v_{s}^{2}} \frac{\partial^{2} \bar{\psi}}{\partial t^{2}} .
\end{aligned}
$$

The last equation applies to each Cartesian component of the vector $\bar{\psi}$.

In Eq. (2.2) $v_{c}$ and $v_{s}$ represent the velocity of dilatational and rotational waves, respectively.

$$
\begin{aligned}
& v_{c}=\left[\frac{2 G(1-\nu)}{\rho_{1}(1-2 \nu)}\right]^{\frac{1}{1}}, \\
& v_{\mathrm{a}}=\left(G / \rho_{1}\right)^{\frac{1}{2}},
\end{aligned}
$$

and $G=$ shear modulus of solid, $\rho_{1}=$ mass density of solid, $\nu=$ Poisson's ratio. Because of the axial symmetric nature of the phenomenon, the vector $\bar{\psi}$ is tangent to a circle of radius $r$, centered on the $z$ axis and parallel with the $x, y$ plane (Fig. 1). Hence

$$
\psi_{x}=-\psi \sin \theta, \quad \psi_{y}=\psi \cos \theta .
$$

Similarly, introducing the radial displacement $R$

$$
U=R \cos \theta, \quad V=R \sin \theta .
$$

The quantities $\phi, \psi$, and $R$ functions only of the coordinates $r$ and $z$. Writing the two Eqs. (2.2) in cylindrical coordinates the first one becomes

$$
\frac{\partial^{2} \phi}{\partial r^{2}}+\frac{1}{r} \frac{\partial \phi}{\partial r}+\frac{\partial^{2} \phi}{\partial z^{2}}=\frac{1}{v_{c}^{2}} \frac{\partial^{2} \phi}{\partial t^{2}}
$$

The second Eq. (2.2), written, for instance, for the component $\psi_{x}$ is

$$
\frac{\partial^{2} \psi_{x}}{\partial r^{2}}+\frac{1}{r} \frac{\partial \psi_{x}}{\partial r}+\frac{1}{r^{2}} \frac{\partial^{2} \psi_{x}}{\partial \theta^{2}}+\frac{\partial^{2} \psi_{x}}{\partial z^{2}}=\frac{1}{v_{s}^{2}} \frac{\partial^{2} \psi_{x}}{\partial t^{2}} .
$$

Introducing the value (2.3) for $\psi_{x}$

$$
\frac{\partial^{2} \psi}{\partial r^{2}}+\frac{1}{r} \frac{\partial \psi}{\partial r}-\frac{\psi}{r^{2}}+\frac{\partial^{2} \psi}{\partial z^{2}}=\frac{1}{v_{s}^{2}} \frac{\partial^{2} \psi}{\partial t^{2}} .
$$

The two Eqs. (2.5) and (2.6) for the unknown scalars $\phi$ and $\psi$ solve the axially symmetric problem. 
The displacement components are expressed by means of $\phi$ and $\psi$. Equations (2.1) become in the present case

$$
\begin{aligned}
& R=\frac{\partial \phi}{\partial r}-\frac{\partial \psi}{\partial z} \\
& W=\frac{\partial \phi}{\partial z}+\frac{\partial \psi}{\partial r}+\frac{\psi}{r}
\end{aligned}
$$

The stress components $\sigma_{r}$ and $\tau$ (Fig. 1) are

$$
\begin{gathered}
\sigma_{r}=2 G\left[\frac{\partial R}{\partial r}+\frac{\nu}{1-2 \nu} \epsilon\right], \\
\tau=G\left[\frac{\partial R}{\partial z}+\frac{\partial W}{\partial r}\right],
\end{gathered}
$$

with

$$
\epsilon=\frac{\partial R}{\partial r}+\frac{R}{r}+\frac{\partial W}{\partial z} \text {. }
$$

The stress components may also be expressed directly in terms of $\phi$ and $\psi$. Substituting (2.7) into (2.8) and taking into account (2.5) and (2.6)

$$
\begin{aligned}
\sigma_{r} & =\rho_{1} b \frac{\partial^{2} \phi}{\partial t^{2}}+2 v_{s}^{2} \rho_{1}\left[\frac{\partial^{2} \phi}{\partial r^{2}}-\frac{\partial^{2} \psi}{\partial r \partial z}\right], \\
\tau & =\rho_{1} \frac{\partial^{2} \psi}{\partial t^{2}}+2 v_{s}^{2} \rho_{1}\left[\frac{\partial^{2} \phi}{\partial r \partial z}-\frac{\partial^{2} \psi}{\partial z^{2}}\right],
\end{aligned}
$$

with

$$
b=\nu / 1-\nu .
$$

The solutions of Eqs. (2.5) and (2.6) for unattenuated waves propagating in the $z$ direction are

$$
\begin{aligned}
& \phi=\phi_{0} K_{0}(m r) \cos (l z-\alpha t) \\
& \psi=\psi_{0} K_{1}(k r) \sin (l z-\alpha t),
\end{aligned}
$$

where $\phi_{0}$ and $\psi_{0}$ are two constants and

$$
\begin{aligned}
& m=l\left(1-\zeta_{2}^{2}\right)^{\frac{1}{2}}, \quad \zeta_{2}=\frac{\alpha}{v_{c} l}=\frac{v}{v_{c}} \\
& k=l\left(1-\zeta_{1}^{2}\right)^{\frac{1}{2},}, \quad \zeta_{1}=\frac{\alpha}{v_{s} l}=\frac{v}{v_{s}}
\end{aligned}
$$

The phase velocity of the waves in the $z$ direction is designated by $v . K_{0}$ and $K_{1}$ are modified Bessel functions of the second kind of zero and first order. These functions are real and vanish exponentially at infinity. Denoting by $D=2 a$ (Fig. 2) the bore diameter, the boundary conditions are

$$
\left.\begin{array}{c}
\tau=0 \\
r=0
\end{array}\right\} \quad \text { at } \quad r=a .
$$

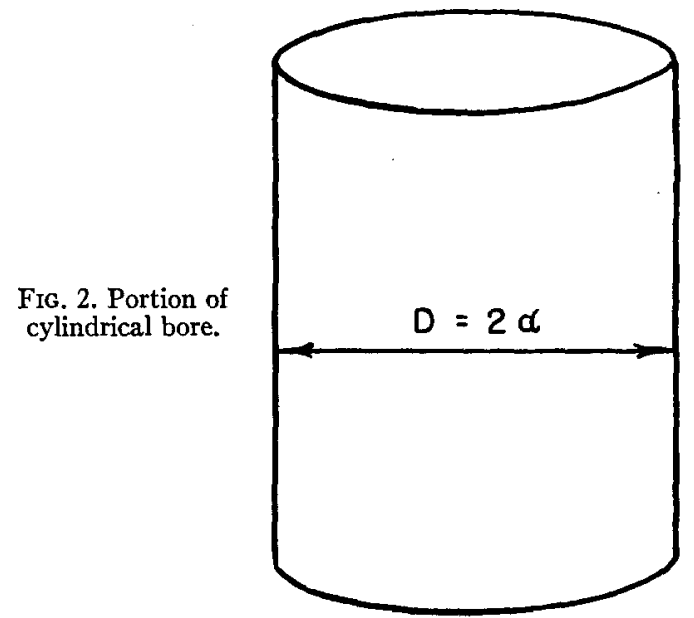

The first boundary condition yields a relation between $\phi_{0}$ and $\psi_{0}$

$$
\psi_{0} K_{1}(k a)\left(2 v_{s}^{2} l^{2}-\alpha^{2}\right)=2 v_{s}^{2} m l \phi_{0} K_{0}^{\prime}(m a)
$$

or

$$
\frac{\phi_{0}}{\psi_{0}}=\frac{\left(2-\zeta_{1}^{2}\right) K_{1}(k a)}{2\left(1-\zeta_{2}^{2}\right)^{1} K_{0}{ }^{\prime}(m a)} .
$$

We use the notation

$$
\begin{aligned}
K_{0}{ }^{\prime}(u) & =\frac{d}{d u} K_{0}(u), \\
K_{0}{ }^{\prime \prime}(u) & =\frac{d^{2}}{d u^{2}} K_{0}(u) .
\end{aligned}
$$

Introducing the second boundary condition $\sigma_{r}=0$ we have

$$
\begin{aligned}
& -\rho_{1} \alpha^{2} b \phi_{0} K_{0}(m a) \\
& +2 v_{s}^{2} \rho_{1}\left[\phi_{0} m^{2} K_{0}^{\prime \prime}(m a)-\psi_{0} k l K_{1}^{\prime}(k a)\right]=0 .
\end{aligned}
$$

This expression may be written in terms of $K_{0}$ and $K_{1}$. We introduce well-known identities satisfied by Bessel functions

$$
\begin{gathered}
K_{0}^{\prime}(u)=-K_{1}(u), \\
K_{0}{ }^{\prime \prime}(u)+\frac{1}{u} K_{0}^{\prime}(u)=K_{0}(u), \\
K_{0}^{\prime \prime}(u)=-K_{1}^{\prime}(u)=\frac{1}{u} K_{1}(u)+K_{0}(u) .
\end{gathered}
$$

With these relations, Eq. (2.14) may be written

$$
\begin{aligned}
-b \zeta_{1}^{2} \phi_{0} K_{0}(m a)+ & 2 \phi_{0}\left(1-\zeta_{2}^{2}\right)\left[\frac{1}{m a} K_{1}(m a)+K_{0}(m a)\right] \\
& +2 \psi_{0}\left(1-\zeta_{1}^{2}\right)^{3}\left[\frac{1}{k a} K_{1}(k a)+K_{0}(k a)\right]=0 .
\end{aligned}
$$




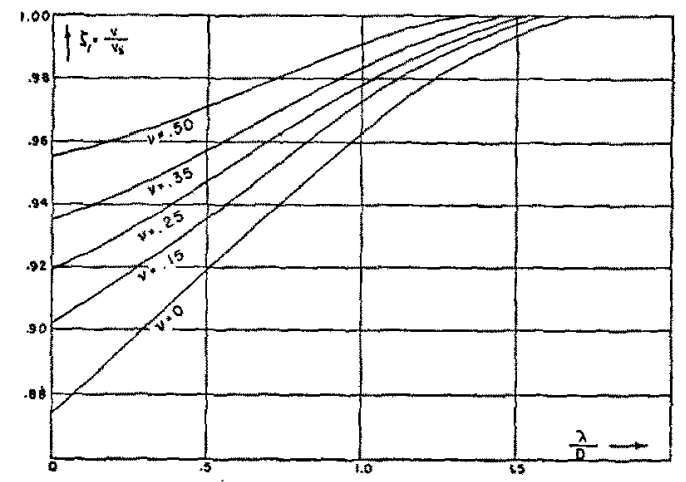

FIG. 3. Phase velocity of surface waves in empty bore.

With the value (2.13) for the ratio $\psi_{0} / \phi_{0}$ this becomes $4\left(1-\zeta_{1}^{2}\right)^{2}\left[\frac{1 !}{k a}+\frac{K_{0}(k a)}{K_{\mathrm{I}}(k a)}\right]$

$$
\begin{aligned}
-2\left(2-\zeta_{1}^{2}\right)\left(1-\zeta_{2}^{2}\right) & {\left[\frac{1}{m a}+\frac{K_{0}(m a)}{K_{1}(m a)}\right] } \\
& +b \frac{\zeta_{1}^{2}\left(2-\zeta_{1}^{2}\right)}{\left(1-\zeta_{2}^{2}\right)^{\frac{1}{2}}} \frac{K_{0}(m a)}{K_{1}(m a)}=0 .
\end{aligned}
$$

Substituting

$$
b=1-2\left[\frac{\zeta_{2}}{\zeta_{1}}\right]^{2}
$$

we find

$$
\begin{aligned}
& 4\left(1-\zeta_{1}^{2}\right)^{3}\left[\frac{1}{k a}+\frac{K_{0}(k a)}{K_{1}(k a)}\right] \\
& -\frac{2\left(2-\zeta_{1}^{2}\right)\left(1-\zeta_{2}^{2}\right)^{\frac{3}{2}}}{m a}-\frac{\left(2-\zeta_{1}^{2}\right)^{2}}{\left(1-\zeta_{2}^{2}\right)^{\frac{1}{3}}} \frac{K_{0}(m a)}{K_{1}(m a)}=0 .
\end{aligned}
$$

Since $m a=l a\left(1-\zeta_{2}^{2}\right)^{2}, k a=l a\left(1-\zeta_{1}^{2}\right)^{1}$, this last equation determines the phase velocity of the axial symmetric surface waves as a function of the Poisson ratio and the variable $l a=(2 \pi a / \lambda)=\pi(D / \lambda)$. Hence the wave shows a dispersion function of the ratio of the wavelength to the diameter of the bore. Physically it must

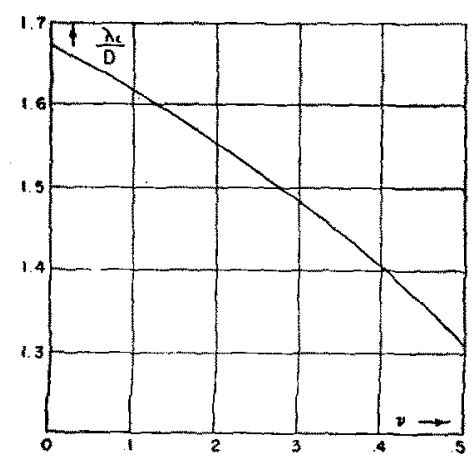

be expected that for very short wavelengths (la large) the velocity must coincide with the velocity of the Rayleigh wave at a plane boundary. This may be verified by putting $l a=\infty$ in Eq. (2.16) for large values of the argument the asymptotic value of the Bessel function is

$$
K_{0}(u)=K_{1}(u)=e^{-u}\left(\frac{\pi}{2 u}\right)^{t}(u \rightarrow \infty) .
$$

Hence in that case Eq. (2.16) reduces to

$$
4\left(1-\zeta_{1}^{2}\right)^{2}-\frac{\left(2-\zeta_{1}^{2}\right)^{2}}{\left(1-\zeta_{2}^{2}\right)^{2}}=0
$$

which coincides with the equation for the Rayleigh wave at a plane boundary. Equation (2.16) has been solved numerically, and a plot of the velocity ratio $v / v_{s}=\zeta_{1}$ as a function of $\lambda / D$ is shown in. Fig. 3 for values of the Poisson ratio $v$ varying between 0 and $\frac{1}{2}$. A significant feature is that all these curves show an increase of the phase velocity $v$ with increasing wave length until $v$ becomes equal to the value $v_{a}$ for the shear wave. The curves are terminated at that point which corresponds to a cut-off wavelength $\lambda_{f}$. For wavelengths larger than this wavelength $\lambda_{c}$ the waves cannot propagate without attenuation. The ratio $\lambda_{c} / D$ depends on the Poisson ratio as follows.

TABLE I.

\begin{tabular}{ll}
\hline$\nu$ & $\lambda_{c} / D$ \\
\hline 0 & 1.670 \\
0.15 & 1.583 \\
0.25 & 1.517 \\
0.35 & 1.445 \\
0.50 & 1.310 \\
\hline
\end{tabular}

The curve $\lambda_{e} / D$ is plotted against $\nu$ in Fig. 4.

The existence of this cut-off wavelength for axial symmetric surface waves is a consequence of two facts: first, that the phase velocity increases with the wavelength, and second, that the radical $\left(1-\zeta_{1}^{2}\right)^{\text {tin }} \mathrm{Eq} .(2.16)$ must be real. Physically these two facts may be understood by bearing in mind that an axial symmetric deformation gives rise to circumferential stresses which do not exist in the two-dimensional case. This causes an apparent increase of rigidity of the material for such symmetric deformations. This rigidity becomes greater as the wavelength increases, that is, as the depth at which the surface waves are felt becomes larger. It is natural therefore that this apparent increase of rigidity causes an increasing value of the phase velocity. This increase, however, cannot occur beyond the point when the velocity of the surface waves becomes larger than the shear-wave velocity, because a disturbance propagating at the surface with such a velocity causes shear waves to be radiated, and the energy of the surface waves would be dissipated.

Finally it is interesting to derive the group-velocity 
curves for such waves. The group velocity $v_{g}$ is

We may write

$$
v_{q}=d \alpha / d l \text {. }
$$

$$
v_{g} / v_{s}=d\left(\zeta_{1} l a\right) / d(l a),
$$

that is, the ratio $v_{g} / v_{g}$ is the derivative of the product $\zeta_{1} l a$ considered as a function of $l a$. The value $v_{g} / v_{g}$ is plotted as a function of $l a=\pi D / \lambda$ in Fig. 5 for different values of the Poisson ratio $\nu$.

\section{PROPAGATION OF WAVES IN A CYLINDRICAL BORE FILLED WITH A FLUID}

We consider the same problem as in the previous section with the addition of a fluid in the bore. We first study the behavior of waves in the fluid independently from the solid. For axially symmetric waves the displacement potential $\phi$ of the fluid satisfies the wave equation expressed in cylindrical coordinates ( $c=$ velocity of sound in the fluid),

$$
\frac{\partial^{2} \phi}{\partial r^{2}}+\frac{1}{r} \frac{\partial \phi}{\partial r}+\frac{\partial^{2} \phi}{\partial r^{2}}=\frac{1}{c^{2}} \frac{\partial^{2} \phi}{\partial t^{2}}
$$

The solution $\phi$ may be written

and

$$
\phi=J_{0}\left[r\left(\frac{\alpha^{2}}{c^{2}}-l^{2}\right)^{\frac{1}{2}}\right] e^{i(l z-\alpha t)} \text { for } \quad \frac{\alpha^{2}}{c^{2}}>l^{2},
$$

$$
\phi=I_{0}\left[r\left(l^{2}-\frac{\alpha^{2}}{c^{2}}\right)^{\frac{1}{2}}\right] e^{i(l z-\alpha t)} \text { for } \frac{\alpha^{2}}{c^{2}}<l^{2} .
$$

$J_{0}$ is the Bessel function of zero order of the first kind and $I_{0}$ the modified Bessel function of zero order of the first kind.

$$
J_{0}(i u)=I_{0}(u) .
$$

The first solution corresponds to conical waves which are reflected at the boundary while the second solution corresponds to Stoneley waves.

The fluid pressure for each case is

$p=-\rho \frac{\partial^{2} \phi}{\partial t^{2}}=\rho \alpha^{2} J_{0}\left[r l\left(\zeta^{2}-1\right)^{\frac{1}{2}}\right] e^{i(l z-\alpha t)}$ for $\zeta>1$,

and ( $\rho=$ mass density of the fluid)

$p=\rho \alpha^{2} I_{0}\left[r l\left(1-\zeta^{2}\right)^{t}\right] e^{i(l z-\alpha t)}$ for $\zeta<1$.

We have put $\zeta=v / c ; v=\alpha / l$ is the phase velocity along the $z$ direction and $c$ is the velocity of sound in the fluid. Denoting the radial displacement of the fluid by

$$
R^{\prime}=\partial \phi / \partial r,
$$

and using the relations

$$
J_{1}(u)=-\frac{d}{d u} J_{0}(u) \quad I_{1}(u)=\frac{d}{d u} I_{0}(u)
$$

we may write for each case

$$
\begin{aligned}
& R^{\prime}=-l\left(\zeta^{2}-1\right)^{\frac{1}{1}} J_{1}\left[r l\left(\zeta^{2}-1\right)^{\frac{1}{2}}\right] e^{i\left(l_{z}-\alpha t\right)}, \\
& R^{\prime}=l\left(1-\zeta^{2}\right)^{\frac{1}{2}} I_{1}\left[r l\left(1-\zeta^{2}\right)^{\frac{1}{2}}\right] e^{i\left(l_{z-\alpha}-\alpha\right.} .
\end{aligned}
$$

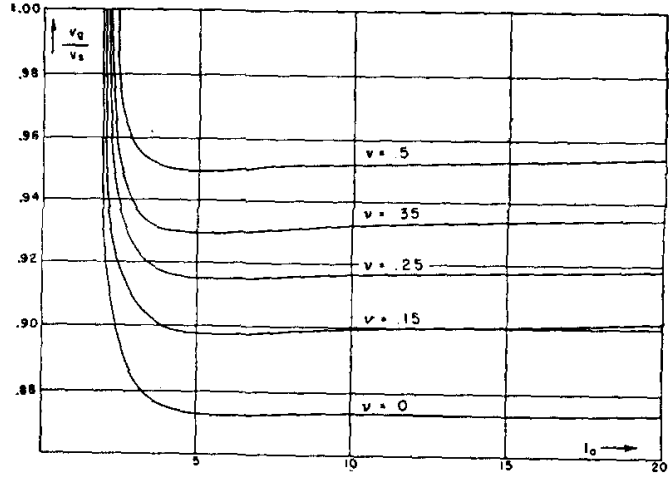

FIG. 5. Group velocity in empty bore.

The corresponding ratios of pressure to fluid displacement at the boundary $r=a$ are therefore

$$
\frac{p}{R^{\prime}}=-\frac{\alpha^{2} \rho}{l} \frac{J_{0}\left[l a\left(\zeta^{2}-1\right)^{1}\right]}{\left(\zeta^{2}-1\right)^{\frac{1}{2}} J_{1}\left[l a\left(\zeta^{2}-1\right)^{\dagger}\right]} \text { for } \zeta>1
$$

and

$$
\frac{p}{R^{\prime}}=\frac{\alpha^{2} \rho}{l} \frac{I_{0}\left[l a\left(1-\zeta^{2}\right)^{\frac{1}{2}}\right]}{\left(1-\zeta^{2}\right)^{\frac{1}{2}} I_{1}\left[l a\left(1-\zeta^{2}\right)^{\frac{1}{2}}\right]} \text { for } \zeta<1 .
$$

If the fluid is contained in a rigid wall, $R^{\prime}=0$ and the corresponding velocities are given by the equation

$$
\left(\zeta^{2}-1\right)^{\frac{1}{2}} J_{1}\left[l a\left(\zeta^{2}-1\right)^{\frac{1}{3}}\right]=0 .
$$

The root $\zeta=1$ corresponds to waves with their plane normal to the axis, while the equation

$$
l a\left(\zeta^{2}-1\right)^{\frac{1}{2}}=\beta_{n}
$$

corresponds to the reflected conical waves. The constants $\beta_{n}$ are the roots $\left(\beta_{n} \neq 0\right)$ of the Bessel function $J_{1}$. For each root, if we plot the velocity $\zeta$ as a function of $\lambda / D(D=2 a, \lambda=$ wavelength) we obtain a hyperbola. Similarly a cylindrical fluid column free at the boundary has reflected conical waves corresponding to the equation

$$
J_{0}\left[\operatorname{la}\left(\zeta^{2}-1\right)\right]=0
$$

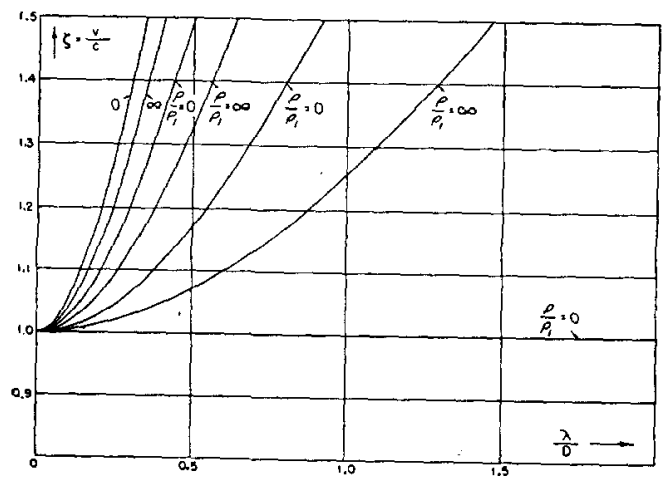

Frg. 6. Phase velocity in fluid for either a rigid wall or the absence of solid wall. 
and again this equation represents a family of hyperbolas.

The branches corresponding to Eq. (3.6) are plotted in Fig. 6 as functions of $\lambda / D=\pi / l a$. They are marked $\rho / \rho_{1}=0$ because they also correspond to the case where the wall is elastic but the fluid has a very small density relative to the solid. The branches corresponding to Eq. (3.8) are represented in the same Fig. 6 and marked $\rho / \rho_{1}=\infty$.

We shall now introduce the effect of the elastic wall. Using equations established in the previous section we derive the ratio of normal stress to displacements at the solid boundary. The stresses in the solid are obtained by substituting expressions (2.10) into (2.9). One of the boundary conditions $r=0$ for $r=a$ is the same as in Sec. 2 and leads to the same relations (2.12) and (2.13) between $\phi_{0}$ and $\psi_{0}$. For the normal stress $\sigma_{r}$ we have at $r=a$

$$
\begin{aligned}
\frac{\sigma_{r}}{2 v_{s}^{2} \rho_{1}}=\left[-\frac{b}{2 v_{s}^{2}} \alpha^{2} \phi_{0} K_{0}(m a)+m^{2} \phi_{0} K_{0}{ }^{\prime \prime}(m a)\right. \\
\left.-k l \psi_{0} K_{1}^{\prime}(k a)\right] \cos (l z-\alpha t) .
\end{aligned}
$$

The radial displacement $R$ is given by (2.7)

$$
R=\left[m \phi_{0} K_{0}{ }^{\prime}(m a)-l \psi_{0} K_{1}(k a)\right] \cos (l z-\alpha t) .
$$

By using Eq. (2.12) we may simplify this to

$$
R l=-\frac{\alpha^{2}}{2 v_{s}^{2}} \psi_{0} K_{1}(k a) \cos (l z-\alpha t) .
$$

If we divide (3.9) by (3.10) and replace $\phi_{0} / \psi_{0}$ by its value (2.13), we get

$$
\begin{aligned}
& \frac{\sigma_{\mathrm{r}}}{v_{\mathrm{s}}^{2} \rho_{1} l R}=\frac{b\left(2-\zeta_{1}^{2}\right)}{\left(1-\zeta_{2}^{2}\right)^{\frac{1}{3}}} \frac{K_{0}(m a)}{K_{0}^{\prime}(m a)} \\
& -\frac{2}{\zeta_{1}^{2}}\left(2-\zeta_{1}^{2}\right)\left(1-\zeta_{2}{ }^{2}\right)^{\frac{K_{0}{ }^{\prime \prime}(m a)}{K_{0}{ }^{\prime}(m a)}}
\end{aligned}
$$

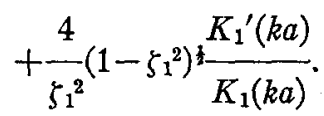

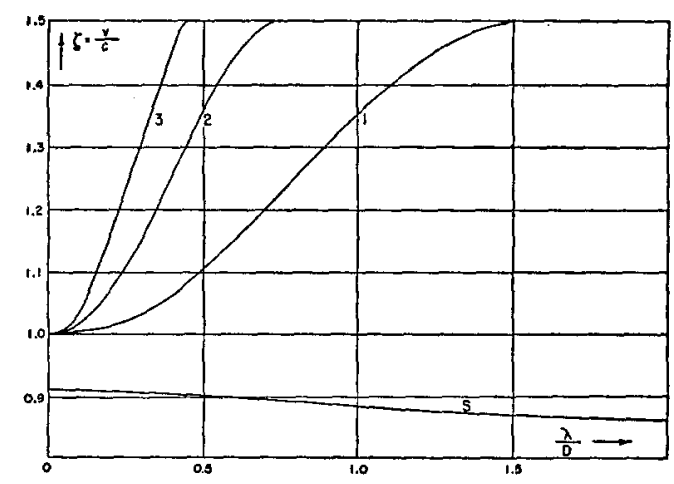

FIG. 7. Phase velocity in fluid-filled bore for $v_{s} / c=1.5, \rho / \rho_{1}=1, \nu=\frac{1}{1}$.
By using identities (2.15) this becomes

$$
\begin{aligned}
\frac{\sigma_{r}}{v^{2}{ }^{2} \rho_{1} l R}= & -\frac{b\left(2-\zeta_{1}^{2}\right)}{\left(1-\zeta_{2}^{2}\right)^{\frac{1}{2}}} \frac{K_{0}(m a)}{K_{1}(m a)} \\
& +\frac{2}{\zeta_{1}^{2}}\left(2-\zeta_{1}^{2}\right)\left(1-\zeta_{2}^{2}\right)^{\frac{3}{3}}\left[\frac{1}{L m a}+\frac{K_{0}(m a)}{K_{1}(m a)}\right] \\
& -\frac{4}{\zeta_{1}^{2}}\left(1-\zeta_{1}^{2}\right)^{\frac{1}{3}}\left[\frac{1}{k a}+\frac{K_{0}(k a)}{K_{1}(k a)}\right] .
\end{aligned}
$$

If we express $b$ as

$$
b=1-2\left[\zeta_{2} / \zeta_{1}\right]^{2},
$$

we may write

$$
\begin{aligned}
\frac{\sigma_{r}}{v_{s}^{2} \rho_{1} l R}=\frac{\left(2-\zeta_{1}^{2}\right)^{2}}{\zeta_{1}^{2}\left(1-\zeta_{2}^{2}\right)^{\frac{1}{2}}} & \frac{K_{0}(m a)}{K_{1}(m a)} \\
+ & \frac{2}{\zeta_{1}^{2}} \frac{\left(2-\zeta_{1}^{2}\right)\left(1-\zeta_{2}^{2}\right)^{\frac{1}{2}}}{m a} \\
& -\frac{4}{\zeta_{1}^{2}}\left(1-\zeta_{1}^{2}\right)^{\frac{1}{2}}\left[\frac{1}{k a}+\frac{K_{0}(k a)}{K_{1}(k a)}\right] .
\end{aligned}
$$

We now introduce the boundary condition at the fluidsolid interface

$$
-p / R^{\prime}=\sigma_{r} / R \text { for } r=a .
$$

From (3.5) and (3.12) this may be written for $\zeta>1$ (reflected waves)

$$
\begin{array}{r}
4\left(1-\zeta_{1}^{2}\right)^{\frac{1}{2}}\left[\frac{1}{k a}+\frac{K_{0}(k a)}{K_{1}(k a)}\right] \\
\frac{2\left(2-\zeta_{1}^{2}\right)^{2}\left(1-\zeta_{2}^{2}\right)^{\frac{1}{2}}}{m a}-\frac{\left(2-\zeta_{1}^{2}\right)^{2}}{\left(1-\zeta_{2}^{2}\right)^{\frac{1}{2}}} \frac{K_{0}(m a)}{K_{1}(m a)} \\
=-\frac{\rho}{\rho_{1}} \frac{\zeta_{1}^{4}}{\left(\zeta^{2}-1\right)^{\frac{1}{3}}} \frac{J_{0}\left[l a\left(\zeta^{2}-1\right)^{\frac{1}{2}}\right]}{J_{1}\left[l a\left(\zeta^{2}-1\right)^{\frac{1}{2}}\right]} .
\end{array}
$$

Similarly for $\zeta<1$ (Stoneley waves)

$$
\begin{aligned}
& 4\left(1-\zeta_{1}^{2}\right)^{7}\left[\frac{1}{k a}+\frac{K_{0}(k a)}{K_{1}(k a)}\right]-\frac{2\left(2-\zeta_{1}^{2}\right)^{2}\left(1-\zeta_{2}^{2}\right)^{\frac{3}{3}}}{m a}-\frac{\left(2-\zeta_{1}^{2}\right)^{2}}{\left(1-\zeta_{2}^{2}\right)^{\frac{1}{2}}} \frac{K_{0}(m a)}{K_{1}(m a)} \\
&=\frac{\rho}{\rho_{1}} \frac{\zeta_{1}^{4}}{\left(1-\zeta^{2}\right)^{\frac{1}{2}}} \frac{I_{0}\left[l a\left(1-\zeta^{2}\right)^{\frac{1}{2}}\right]}{I_{1}\left[l a\left(1-\zeta^{2}\right)^{\frac{1}{2}}\right]} .
\end{aligned}
$$

The two Eqs. (3.13) and (3.14) determine the dispersion of the waves in the fluid-solid system. The functional relationship of these equations becomes clear if we recall the significance of the symbols. Consider $\zeta$ as the unknown variable

$$
\zeta=v / c, \quad \zeta_{1}=c \zeta / v_{s}, \quad \zeta_{2}=v_{s} \zeta_{1} / v_{c} .
$$




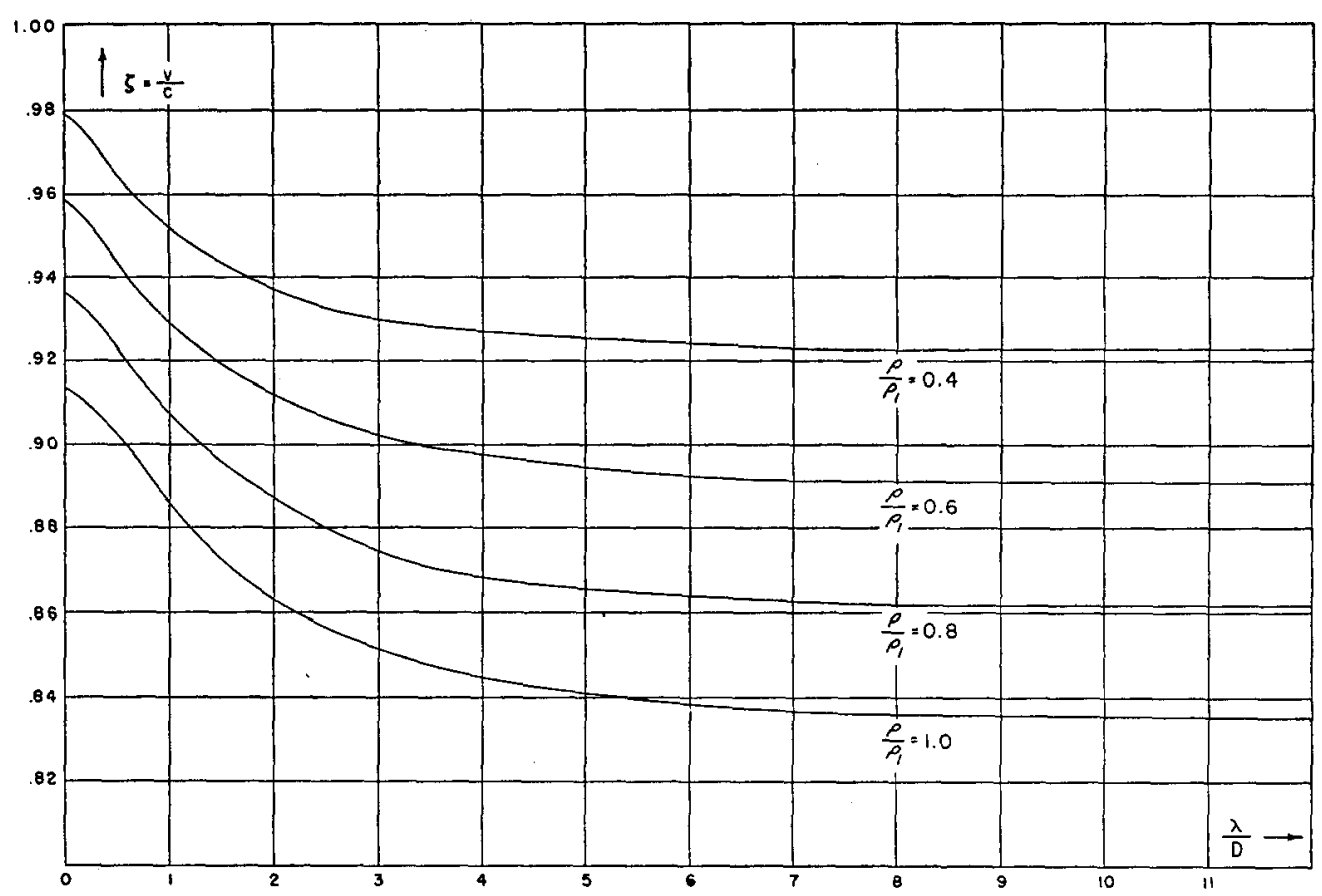

FIG. 8. Phase velocity for Stoneley type wave for various density ratios (branch marked $S$ in Fig. 7).

Hence $\zeta_{1}$ and $\zeta_{2}$ are functions of $\zeta$ through the parameters $v_{s} / c$ and $v_{s} / v_{c}$. The parameter $v_{s} / v_{c}$ is a function of the Poisson ratio $\nu$ only.

$$
v_{c} / v_{s}=2(1-\nu) / 1-2 \nu \text {. }
$$

When $\nu$ varies from zero to $0.5, v_{c} / v_{8}$ varies from $\sqrt{2}=1.414$ to $\infty$. Also we remember that

$$
k a=l a\left(1-\zeta_{1}^{2}\right)^{\frac{1}{2}}, \quad m a=l a\left(1-\zeta_{2}^{2}\right)^{\frac{1}{2}} .
$$

Therefore Eq. (3.13) and (3.14) may be considered as determining implicitly the value of $\xi=v / c$ as a function of the wavelength variable $l a=\pi D / \lambda$ for given values of the parameters $v_{s} / c, \rho / \rho_{1}, \nu$. These three parameters which characterize the fluid-solid system determine the nondimensional dispersion curves of the waves. Plots have been derived from these equations and will now be discussed.

Figure 7 is a plot of the dispersion curves $\zeta=v / c$ as a function of $\lambda / D(\lambda=$ wavelength, $D=$ diameter of the bore) for the following particular values of the characteristic parameters:

$$
v_{s} / c=1.5, \quad \rho / \rho_{1}=1, \quad \nu=1 / 4 .
$$

These curves show the same qualitative behavior as the curves for waves in the fluid alone, plotted in Fig. 6 . The horizontal line of Fig. 6 which corresponded to plane waves becomes branch $S$ in Fig, 7 and represents Stoneley waves propagating with a velocity lower than the velocity of sound in the fluid. The branches marked $1,2,3$ correspond to the reflected waves. There are an infinite number of such branches and only three of them have been represented.
Figure 8 is a plot of the Stoneley wave branch $S$ of the dispersion curve to exhibit the influence of the density ratio $\rho / \rho_{1}$. The value of $\zeta=v / c$ is plotted as a function of $\lambda / D$ for

$$
v_{s} / c=1.5, \quad \nu=\frac{1}{4},
$$

and four values of $\rho / \rho_{1}$ :

$$
\rho / \rho_{1}=0.4,0.6,0.8,1.0 \text {. }
$$

The velocity tends toward a horizontal asymptote for large $\lambda / D$. The asymptotic value is found to be

$$
\begin{aligned}
& \frac{v}{c}=\frac{1}{\left[1+\left(\rho c^{2} / \rho_{1} v_{s}^{2}\right)\right]^{\frac{1}{2}}} \\
& \frac{v}{c}=\frac{1}{\left[1+\left(\rho c^{2} / G\right)\right]^{\frac{1}{3}}} .
\end{aligned}
$$

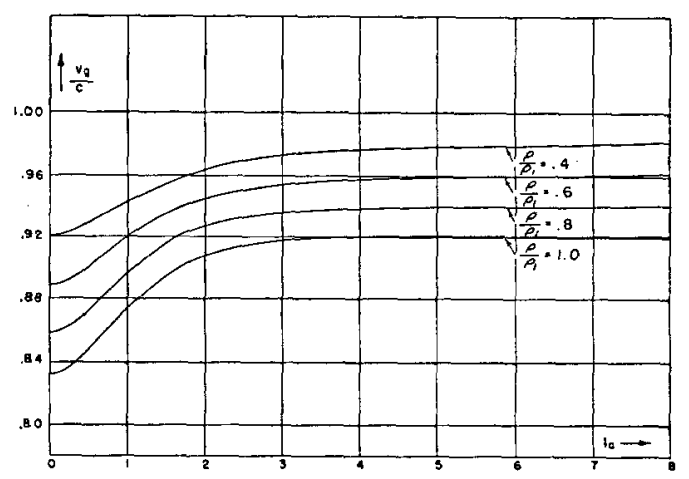

Fic. 9. Group velocity for Stoneley type waves of Fig. 8 . 


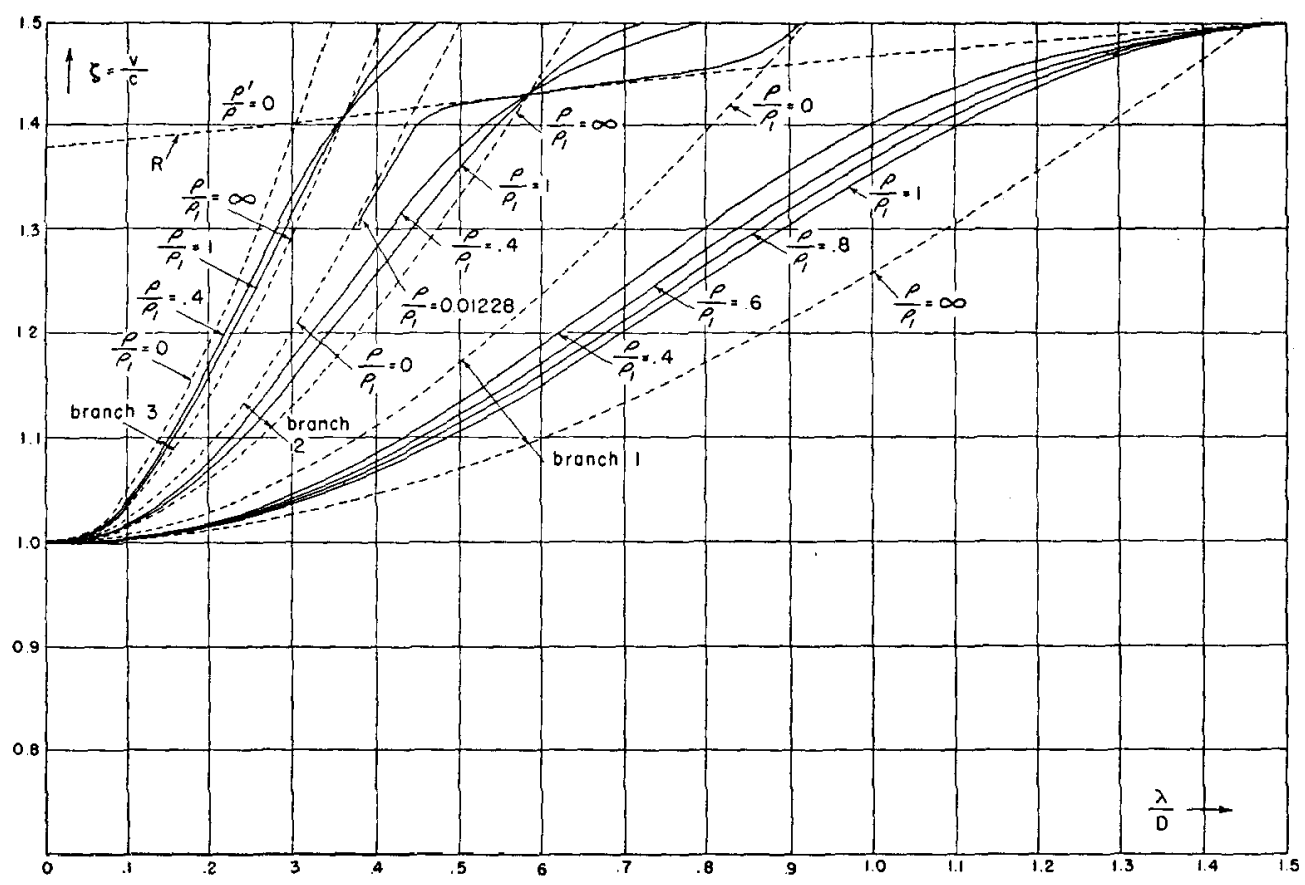

Fig. 10. Phase velocity of reflected type waves for various density ratios (branches marked 1, 2, 3, etc., in Fig. 7).

This formula is valid for all values of $\rho / \rho_{1}$ and $\nu$. It shows that the asymptotic value depends only on a single parameter $\rho c^{2} / G$ which is the ratio of the compression rigidity of the fluid $\rho c^{2}$ to the shear modulus $G$ of the solid. It will be noted that the phase velocity becomes practically independent of the wavelength $\lambda$ for ratios $\lambda / D$ of the order of 5 . It may be said that for $\lambda / D>5$ we are in a region where the Stoneley waves become identical with those studied in the classical theory of the water-hammer phenomenon. For small values of the wavelength, the velocity coincides with the Stoneley wave velocity at a plane interface of two semi-infinite media.

Figure 9 is a plot of the group-velocity curves corresponding to the Stoneley waves of Fig. 8. Denoting by $v_{0}$

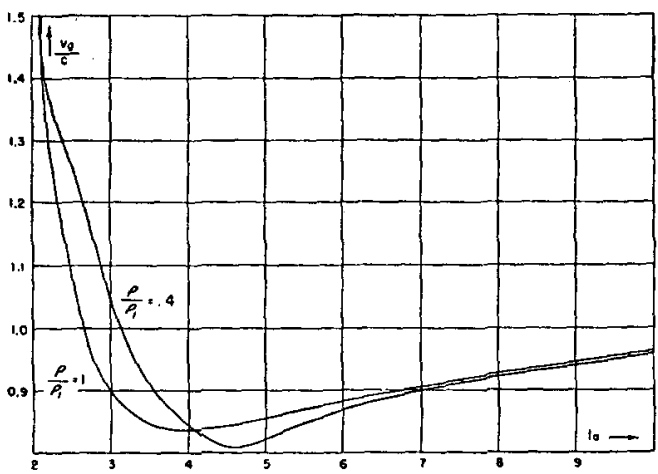

FIG. 11. Group velocity for reflected type wave of branch 1 of Fig. 10 for two density ratios. the group velocity

$$
\frac{\nu_{0}}{c}=\frac{d(\zeta l a)}{d(l a)}
$$

The derivative is taken with respect to $l a=\pi D / \lambda$ considered as the independent variable. The value $v_{a} / c$ is plotted versus la for four values of $\rho / \rho_{1}$ (density ratio of fluid to solid).

Figure 10 shows a series of phase-velocity curves for the reflected wave branches. The value $v / c$ is plotted versus $\lambda / D$ for the same particular values of the parameters, $v_{s} / c$ and $\nu$, namely,

$$
v_{s} / c=1.5, \quad \nu=\frac{1}{4} .
$$

The first branch is plotted for four values of the

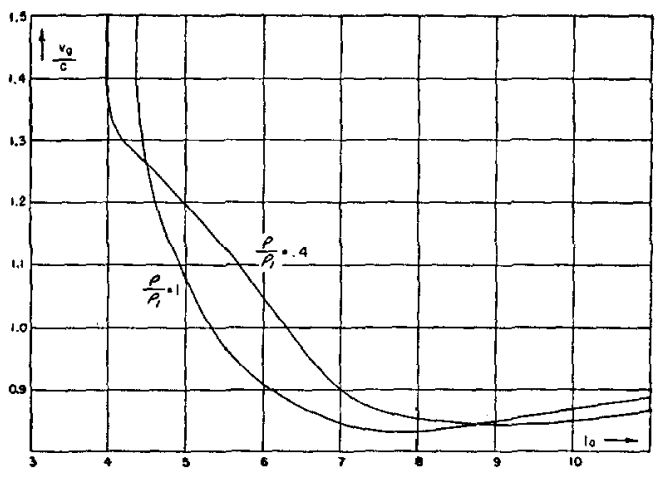

FIG. 12. Group velocity for reflected type waves of branch 2 of Fig. 10 for two density ratios. 
parameter

$$
\rho / \rho_{1}=0.4,0.6,0.8,1.0 \text {. }
$$

The second and third branches are plotted for two values

$$
\rho / \rho_{1}=0.4 \text { and } 1.0 \text {. }
$$

The second branch is plotted also for the value $\rho / \rho_{1}$ $=0.01228$ in order to show how the curve approaches the case $\rho / \rho_{1}=0$ when $\rho / \rho_{1}$ becomes very small. The extreme cases $\rho / \rho_{1}=0$ and $\rho / \rho_{1}=\infty$ are plotted as dotted lines. They are made up of the same hyperbolas as in Fig. 6 and of the surface-wave dispersion curve marked $R$. This surface wave is nothing but a Rayleigh wave of axial symmetry. Its dispersion curve $R$ is the same as the surface-wave dispersion curve for $\nu=\frac{1}{4}$

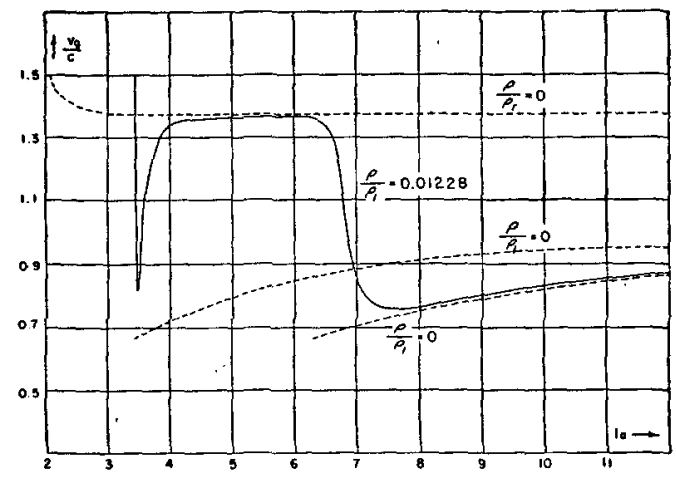

Fig. 13. Group velocity for branch 2 of Fig. 10 for the case of a small fluid density.

plotted in Fig. 3, for the case of an empty bore. The case $\rho / \rho_{1}=0.01228$ illustrates how, for small values of the density ratio, the dispersion branches tend to follow two successive hyperbolas $\rho / \rho_{1}=0$ and the intersecting curve $R$ for the pure surface wave. Whatever the value of $\rho / \rho_{1}$ all branches pass through the intersections of the $R$ curve with the hyperbolas $\rho / \rho_{1}=\infty$.

Figure 11 shows group-velocity curves for the first branch of the reflected waves of Fig. 10. The ratio $v_{g} / c$ is plotted versus $l a$ for the two cases $\rho / \rho_{1}=0.4$ and 1.00 . Also $v_{s} / c=1.5, \nu=\frac{1}{4}$. Note the existence of a minimum value of the group velocity.

Figure 12 shows group-velocity curves for the same cases as in the previous Fig. 11, but for the reflected waves of the second branch.

Figure 13 shows the group-velocity curve for the

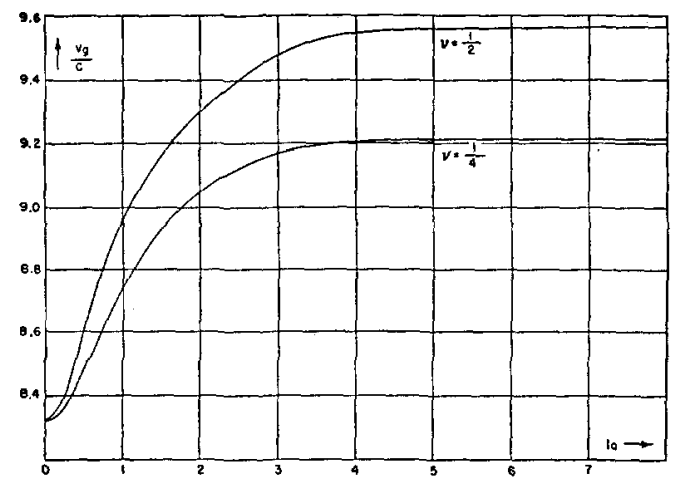

FiG. 14. Influence of Poisson's ratio on the group velocity of the Stoneley type waves.

second branch of Fig. 10 in the case $\rho / \rho_{1}=0.01228$. It is seen that for small values of $\rho / \rho_{1}$ the curve tends to follow either one of the three group-velocity curves corresponding to the case $\rho / \rho_{1}=0$ which are shown as dotted lines. The dotted curve on top of the figure is the group velocity for the Rayleigh wave in the empty bore, while the two lower ones are group velocities for the reflected fluid waves between rigid boundaries.

Figure 14 illustrates the influence of the Poisson ratio $\nu$ on the group velocity of Stoneley waves for the case $v_{s} / c=1.5, \rho / \rho_{1}=1$. Two plots of $v_{g} / c$ versus la are shown for $\nu=\frac{1}{2}$ and $\nu=\frac{1}{4}$.

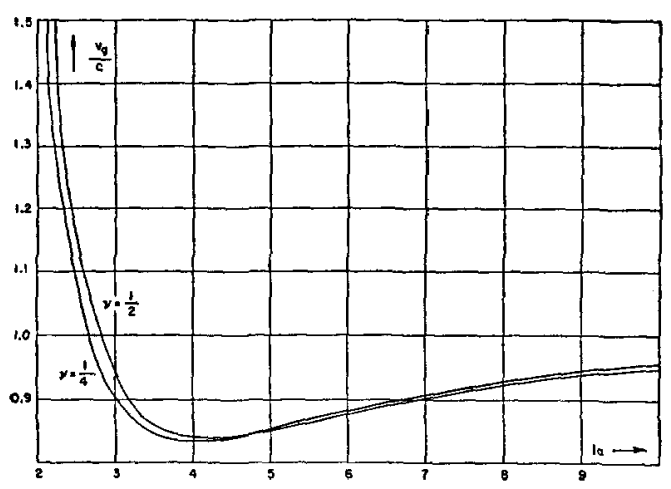

FIg. 15. Influence of Poisson's ratio on the group velocity of reflected type waves of branch 1 .

Figure 15 illustrates the influence of the Poisson ratio $\nu$ on the group velocity of the first branch of the reflected waves for the case $v s / c=1.5, \rho / \rho_{1}=1$. Two plots of $v_{g} / c$ versus la are shown for $\nu=\frac{1}{2}$ and $\nu=\frac{1}{4}$. 